\title{
Responses to exogenous gonadotrophin treatment in contemporary weaned sows
}

\author{
J. Patterson', A. Cameron', T. Smith', A. Kummer', R. Schott², L. Greiner ${ }^{3}$, \\ J. Connor ${ }^{3}$ and G.R. Foxcroft'
}

'Swine Reproduction-Development Program, Swine Research \& Technology Centre, University of Alberta, Edmonton, AB, T6C 2P5 Canada; ${ }^{2}$ PIC North America, 100 Bluegrass Commons Boulevard, Suite 2200, Hendersonville, TN 37075; ${ }^{3}$ Innovative Swine Solutions, LLC, PO Box 220, Carthage, II.

62321, USA

The principal goal of commercial breeding herds is to consistently meet weekly breeding targets. Weaned sows failing to return to estrus within $7 \mathrm{~d}$ after weaning contribute to missed breeding targets and increased non-productive sow days. Treatment with low doses of exogenous gonadotrophins (GT) has traditionally been used to advance and synchronize estrus in weaned primiparous sows. However, in well managed contemporary commercial sow farms, more than $90 \%$ of sows may return to estrus within $7 \mathrm{~d}$ after weaning, posing questions about the likely efficacy of exogenous GT treatment. Therefore, the primary objective of the present study was to determine the response to $\mathrm{GT}$ treatment at weaning in contemporary parity 1 commercial sows with lactation lengths typical of the North American swine industry.

Primiparous crossbred sows (PIC C22, $n=275$; and PIC C29, $n=131$ ) from a 5,000 sow commercial farrow-to-wean facility (Wildcat, Carthage Veterinary Service, Carthage, IL) were blocked by estimated farrowing weight and genetic line and then randomly allocated to either receive a combination dose of $400 \mathrm{IU}$ eCG and $200 \mathrm{IU}$ hCG (PG600, Intervet, USA, De Soto, KS) I.M. in the neck on the morning of weaning (PG group; $n=189$ ), or to be untreated controls (CON group; $n=218$ ). From the day after weaning, all sows were provided twice daily fence-line contact with mature boars for stimulation and detection of estrus. Sows were bred according to established herd protocols with doses of $3.0 \times 10^{9} \mathrm{sperm}$ cells/insemination. Reproductive parameters analyzed were estrus synchronization rate (ESR), determined as the number of sows with observed standing heat within $7 \mathrm{~d}$ after weaning, weaning-to-estrus interval (WEI), proportion of sows bred over a 3-d period (BRD3), proportion of sows bred that farrowed (FR), total litter size (TB), and total live born piglets (BA) at farrowing. Based on WEI, sows were retrospectively grouped into 4 categories (WCAT); 1) Target Breed Week: sows bred with in 7 d post-weaning, 2) "Tail-enders" sows with an extended 8-19 d WEI , 3) "Missed heats" sows detected in heat $\geq 20 \mathrm{~d}$ post weaning, or 4) No detected heat by $30 \mathrm{~d}$ post weaning.

Estimated farrowing $(193.6 \pm 1.5 \mathrm{vs} 192.2 \pm 1.6 \mathrm{~kg})$ and weaning $(189.4 \pm 1.3 \mathrm{vs} 188.0 \pm 1.4 \mathrm{~kg})$ weights were similar in CON and PG sows, respectively, and marginal weight loss in lactation was recorded across all sows $(4.2 \mathrm{~kg})$. Considering data from all sows assigned to treatment (Table 1$)$, treatment did not affect the proportion of sows bred within $7 \mathrm{~d}$ after weaning (ESR), or within a 3-d breeding window (BRD3). However, the timing of this 3 -d breeding window ( $d 4,5$ and 6 for CON vs $\mathrm{d} 3,4$ and 5 for $P G$ sows) reflected a shorter ( $P<0.001)$ WEI in PG compared to CON sows. PG treatment also eliminated the incidence of sows with an extended WEI (classified as "tail enders"), and reduced variance $(P<0.05)$ in WEI in PG $(0.07)$ compared to CON $(0.14)$ sows when considered over the 19-d period after weaning. For those sows bred within $7 \mathrm{~d}$ after weaning, farrowing rate $(82.8$ vs $86.4 \%)$, total born (12.2 \pm .3 vs $12.9 \pm .3)$ and born alive $(11.6 \pm .3$ vs $12.1 \pm .3)$ were 
not different $(P>0.05)$ between $P G$ and $C O N$ sows, respectively, and a combined Fertility index (as defined in Table 1) indicated no gain in productivity in response to GT treatment.

Consistent with earlier reports (Kirkwood et al. 1999; Knox et al. 2001; Vargas et al. 2006), exogenous GT treatment in the present study shortened the WEI, but as also reported by Bates et al. (2000) had no effect on estrous synchronization rate, farrowing rate or subsequent litter size born. These results contrast earlier reports of a negative relationship between a shorter WEI and subsequent litter size born (Kirkwood et al.. 1999; Knox et al. 2001; Vargas et al. 2006). These differences in the response to GT treatment may reflect the very synchronous return to estrus seen in the Control sows and good lactational management as reflected in the marginal overall loss in estimated sow body weight at weaning.

Table 1. Effects of treatment (T; exogenous $G T$ at weaning (PG) or untreated controls (CON)), genotype (G), and their interaction on the distribution of observed estrus in all sows on experiment, and productivity of those sows bred within 7 days after weaning.

\begin{tabular}{|c|c|c|c|c|c|}
\hline \multirow[t]{2}{*}{ Treatment } & \multirow[t]{2}{*}{ CON } & \multirow[t]{2}{*}{ PG } & \multicolumn{3}{|c|}{ P-Value of effect } \\
\hline & & & $T$ & $\mathrm{G}$ & $\mathrm{T} \times \mathrm{G}$ \\
\hline Sows assigned (n) & 218 & 189 & & & \\
\hline ESR $(\%)$ & 88.1 & 92.1 & 0.22 & 0.07 & 0.38 \\
\hline WEI (d) & $4.5 \pm .07$ & $4.0 \pm .07$ & 0.001 & 0.42 & 0.88 \\
\hline BRD3 (\%) & 79.2 & 85.2 & 0.27 & 0.34 & 0.13 \\
\hline Sows farrowed (n) & 166 & 144 & & & \\
\hline$F R(\%)$ & 86.5 & 82.8 & 0.71 & 0.28 & 0.32 \\
\hline TB & $12.9 \pm .3$ & $12.2 \pm .3$ & 0.11 & 0.45 & 0.24 \\
\hline $\mathrm{BA}$ & $12.1 \pm .3$ & $11.6 \pm .3$ & 0.20 & 0.38 & 0.22 \\
\hline \multirow[t]{2}{*}{ Fertility index' } & 983 & 930 & & & \\
\hline & CON & PG & $T$ & WCAT & $T \times$ WCAT \\
\hline $\begin{array}{l}\text { Sows per WEI category } \\
(1 / 2 / 3 / 4) \%\end{array}$ & $88.1 / 2.8 / 2.3 / 6.9$ & $92.1 / 0.5 / 3.1 / 4.2$ & 0.34 & 0.001 & 0.14 \\
\hline
\end{tabular}

' Fertility index $=$ sows bred in $<7$ days after weaning and farrowed per 100 sows weaned $x$ TB

The absence of sows classified as "tail enders" in the PG group may be of practical significance. Assuming that the incidence of "tail enders" will increase at more adverse times of year due to seasonal effects on lactation feed intake and overall reproductive performance, the apparent ability of GT treatment to induce an early ovulation in these sows is of interest. Those GT treated sows showing a silent first heat and then detected in estrus at 19 or more days after weaning will have active corpora lutea at days 17-19 after weaning. These sows should therefore respond to luteolytic prostaglandin treatment and could then either be bred if they show a clear standing estrus or be reliably culled as either showing persistent silent heats or as being totally anovulatory. The return on investment of being able to impose a standardized protocol for reaching a culling decision based on reliable indicators of reproductive performance after weaning, and thus reduce unwarranted non-productive sow days seen in many herds, may be substantial.

\section{References}

Bates RO, Kelpinski J, Hines B \& Ricker D 2000 Hormonal therapy for sows weaned during fall and winter. Journal of Animal Science 78 2068-2071.

Kirkwood RN 1999 Pharmacological intervention in swine reproduction. Swine Health Production 7 29-35.

Knox, RV, Rodriguez-Zas SL, Miler GM, Willenburg KL \& Robb JA. 2001 Administration of P.C. 600 to sows at weaning and the time of ovulation as determined by transrectal ultrasound. Journal of Animal Science 79 796-802.

Vargas A], Bernardi ML, Wentz I, Borchardt Neto G \& Bortolozzo F. 2006 Time of ovulation and reproductive performance over three parities after treatment of primiparous sows with PG600. Theriogenology 66 2017-2023. 